\title{
La sombra del sahuaro
}

\author{
Faustino Sánchez Garduño ${ }^{1}$ Jorge A. Castillo $^{3}$ \\ Pedro Miramontes ${ }^{1,2}$ \\ ${ }^{1}$ Facultad de Ciencias, Universidad Nacional Autónoma de México \\ ${ }^{2}$ Institute for Interdisciplinary Research. Biocomputing Group, University of Leipzig \\ ${ }^{3}$ Unidad Académica de Matemáticas de la Universidad Autónoma de Guerrero, campus Acapulco. \\ e-mail: pedro@bioinf.uni-leipzig.de
}

\begin{abstract}
Resumen
En este ensayo se describen algunas regularidades morfológicas que existen en la arquitectura de las plantas. Con el propósito de emprender su modelación matemática, se estudian sus regularidades geométricas basadas en la sucesión de Fibonacci y la sección áurea. Se muestra el modelo morfogenético de Alan M. Turing y se presenta un modelo que reproduce la forma de una clase de cactáceas.
\end{abstract}

\section{Introducción}

Para el pueblo pápago, que se nombran a sí mismos o'ódham, el sahuaro o saguaro, hash'an, en su lengua, es una planta que les proporciona frutos para la producción de jarabes y vino. Cuenta su leyenda, que una muchacha alta y bella llamada Sugu-ik, fue inesperadamente hundiéndose en la tierra ante la desesperación de su madre y conocidos. Luego que desapareció, su madre colocó comida y agua en el sitio donde se le vio por última vez pensando en que quizá por ahí rondara su espíritu y necesitara sustento. Un día la madre regresó para depositar su carga y se encontró que en el lugar donde su hija se hundió había surgido un bello y enorme saguaro.

Los saguaros son plantas nativas del desierto de Sonora que comprende la parte norte del estado del mismo nombre y la región sur de Arizona. Es apreciado como material de construcción y fuente de alimentos tanto por los pápagos como por los seris. En el orden taxonómico pertenecen a la familia de la cactáceas, que se caracterizan por la presencia genérica de espinas y tres tipos de tallos: los aplanados, como los nopales, el columnar, como los saguaros, y el globoso, como las biznagas. Estas plantas tienen flores muy vistosas y frutos ricos en azúcares que sirven de alimento a una buena variedad de la fauna del desierto.

Las cactáceas globosas y columnares tienen una arquitectura muy peculiar: sus tallos tienen estrías horizontales que en ocasiones se fusionan y en otras se bifurcan. En la figura 1 se puede apreciar una especie de biznaga como ejemplo de las primeras y un saguaro de las segundas.

Desde el punto de vista de la ciencia, una pregunta obligada es: ¿por qué tienen precisamente esa forma las cactáceas? Esta pregunta, y su generalización a la forma de todos los 


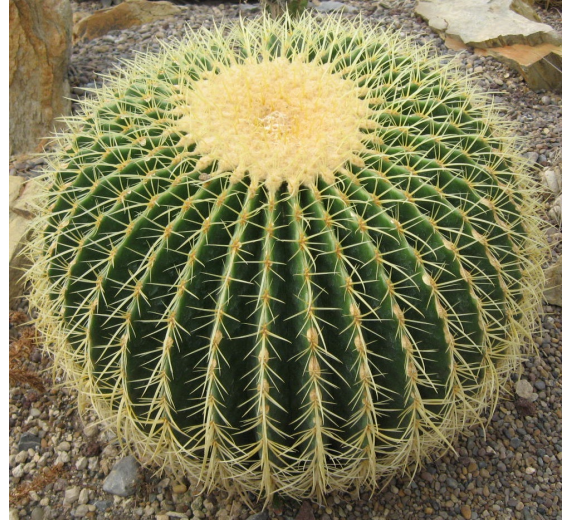

(a) Biznaga

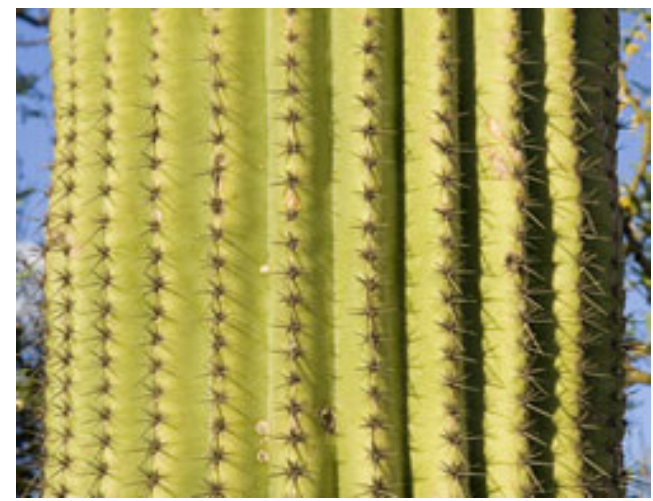

(b) Saguaro

Figura 1: Cactáceas. Se puede observar que, aunque la biznaga es globular y el saguaro columnar, sus costados son esencialmente iguales: estrías o costillas verticales, en un caso sobre una esfera, y en el otro sobre un cilindro

seres vivos, es motivo de gran controversia y agrias disputas entre varias escuelas del pensamiento. En este ensayo, vamos a mostrar que existe una línea de estudio que puede explicar la forma de las cactáceas a partir de primeros principios y, por lo tanto, al matematizar el objeto de la argumentación, se le quita peso a la opinión personal pues sus resultados son matemáticamente demostrables.

Las extraordinarias regularidades geométricas en las plantas se conocen desde tiempos inmemoriales y el estudio sistemático de la manera en que se disponen espacialmente las hojas y tallos se llama Filotaxia. Estas regularidades no escaparon a la atención de los filósofos griegos. Uno de los primeros tratados escritos en donde se describe la forma de las plantas y que ha sobrevivido el paso del tiempo es Sobre las causas de la plantas del discípulo y heredero intelectual de Aristóteles, Teofrasto de Ereso. Posteriormente, Plinio el Viejo, describe en su Historia natural los patrones filotácticos de las plantas. Sin embargo, tuvieron que transcurrir diez y siete siglos para que la filotaxia se integrara como parte legítima de la botánica gracias a la obra del naturalista suizo Charles Bonnet Recherches sur l'usage des feuilles dans les plantes en la cual, por cierto, propone que las plantas tienes capacidades sensoriales e incluso de discernimiento. Los patrones filotácticos que más han llamado la atención son aquellos en los que es evidente la geometría espiral basada en la sucesión de Fibonacci y en la proporción áurea. De ellas hablaremos en las siguientes secciones.

\section{La filotaxia}

Una mirada rápida al jardín, al huerto o al puesto de frutas en el mercado, permite encontrar las regularidades geométricas que mencionamos. Un vistazo a la figura 2 nos muestra tres ejemplos de plantas que hemos visto muchas veces pero que quizá nunca nos hayamos detenido a observarlas cuidadosamente. La figura 2a nos muestra el muy familiar girasol. En el extremo de su tallo se encuentra la terminal capitular, lo que normalmente pensamos que 


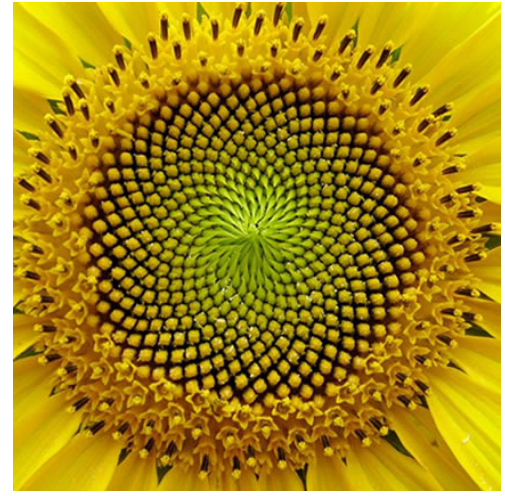

(a) Girasol

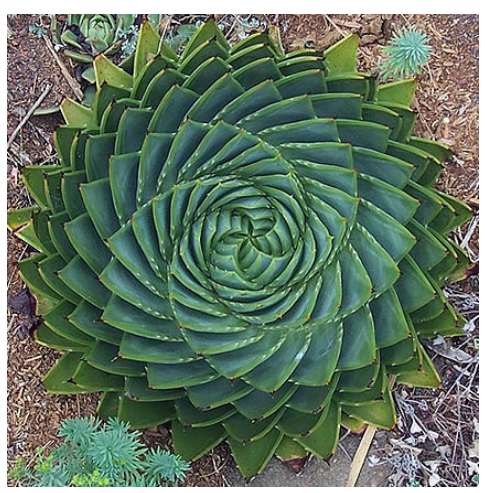

(b) Sábila

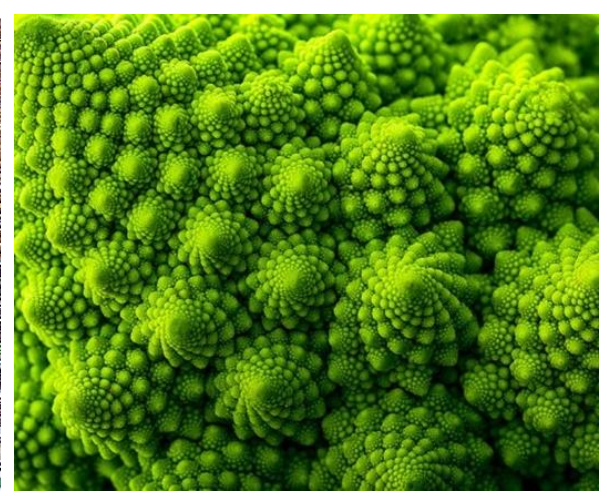

(c) Broccoli romanesco

Figura 2: Tres ejemplos de filotaxia

es la flor, cuyo disco interior está formado por muchas florecitas. En la figura mencionada se ve que dichas florecitas no están dispuestas al azar. Todo lo contrario, la regularidad geométrica de su arreglo. En el extremo del tallo se encuentra la terminal capitular, lo que normalmente pensamos que es la flor, cuyo disco interior está formado por muchas florecitas. En la figura mencionada se ve que dichas florecitas no están dispuestas al azar. Todo lo contrario, la regularidad geométrica de su arreglo espacial es asombrosa: se aprecia que las florecitas forman filas dispuestas como espirales que se abren a la derecha y que se traslapan con otras que se abren a la izquierda. Si el lector tiene la paciencia de contar llegará a la conclusión de que hay 34 espirales que se abren en la dirección de las manecillas del reloj y 21 a contrarreloj. El girasol pertenece a la familia de las asteráceas, entre las cuales se encuentran la manzanilla, la margarita y la achicoria. Dicha familia tiene cerca de veinticuatro mil especies pero todas tiene en común la terminal capitular y las inflorescencias en el disco central. Todas ellas tienen la misma geometría pero en algunas los números de espirales hacia un lado o hacia el otro van como 21-13 o 13-28 y a veces 55-34. El hecho es que siempre el número de espirales hacia un lado y hacia el otro son números consecutivos de la sucesión de Fibonacci.

Recordemos que la sucesión de Fibonacci que se define como:

$$
F_{n}=F_{n-1}+F_{n-2} \text { con } F_{0}=1 \text { y } F_{1}=1
$$

cuyos primeros términos son:

$$
1,1,2,3,5,8,13,21,34 \ldots
$$

En la figura 2b se aprecia el mismo fenómeno pero se puede agregar una dimensión adicional: las hojas de la sábila se van enroscando a lo largo de espirales mientras rotan alrededor de un centro común que es el tallo de la planta. En la figura 3 se representa de manera simplista el tallo de una planta y los brotes (hojas) a lo largo de él. La curva imaginaria que une a las hojas sucesivas de abajo hacia arriba (de las más antiguas a las más recientes) se llama parastiquia mientras que una línea imaginaria, paralela al eje del cilindro 


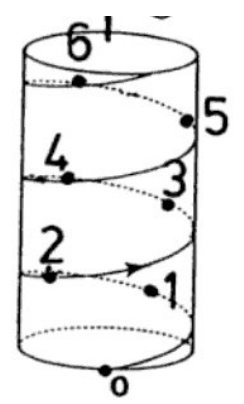

Figura 3: Representación simbólica de la filotaxia en los tallos y brotes. El cilindro representa un tallo, los puntos numerados en sitio de un brote y la línea que los une es una parastiquia

que une un brote con otro en su camino se llama ortostiquia. Se define el Índice Foliar, $\mathbb{F}$, de una planta como el cociente:

$$
\mathbb{F}=\frac{N_{h}}{N_{v}}
$$

donde $N_{h}$ y $N_{v}$ son, respectivamente, el número de brotes que se encuentran a lo largo de una parastiquia que une a dos brotes sobre la misma ortostiquia. Una inspección en el campo nos va a llevar a la conclusión de que hay muchas plantas con índice foliar de $2 / 1$, llamado dístico, en ellas los brotes se encuentran opuestos y sobre un mismo plano (un helecho, por ejemplo). El índice decusado $3 / 1$ es aquel en el cual los brotes se encuentran opuestos uno con otro y a noventa grados de la pareja siguiente como en, ya lo habrán adivinado, la hierbabuena. Podemos seguir con el índice 5/2 llamado tresbolillo y es mucho muy común en la naturaleza (buena parte de los rosales y el girasol), el 8/3, llamado octastiquio (el papayo, ciruelo y muchos frutales). De seguir, encontraremos que las plantas tienen siempre alguno de los índices foliares:

$$
\frac{2}{1}, \frac{3}{1}, \frac{5}{2}, \frac{8}{3}, \frac{13}{5} \ldots
$$

Donde se puede apreciar que los índices foliares son cocientes de números de Fibonacci pero no consecutivos sino de la forma $\frac{F_{n+2}}{F_{n}}$. Dada la definición de la sucesión de Fibonacci, es fácil comprobar que el cociente anterior tiene la forma:

$$
\frac{F_{n+2}}{F_{n}}=\frac{F_{n+1}+F_{n}}{F_{n}}=1+\frac{F_{n+1}}{F_{n}}
$$

Expresión que utilizaremos más adelante.

Por otra parte y para cerrar esta sección, vale la pena comentar que el brócoli romanesco (figura 2c) técnicamente es una yema florífera de una planta muy cercana a la coliflor. Aquí también se aprecian las espirales que se abren a reloj y a contrarreloj pero, adicionalmente, se ilustra un rasgo muy apreciado por biólogas y matemáticos: la autosemejanza. El brócoli 

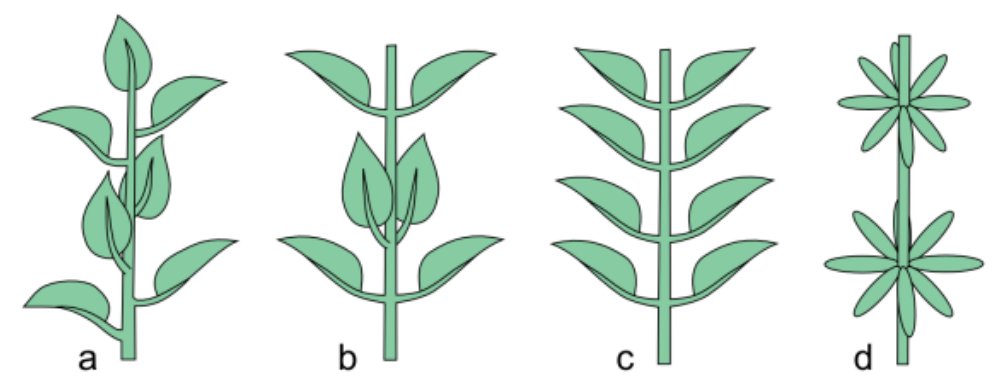

Figura 4: Ejemplos de índices foliales: a) tresbolillo, b) decusado, c) dístico y d) verticilado

romanesco está formado de protuberancias con geometría de Fibonacci, que están hechas de protuberancias, que están hechas de protuberancias...

\section{La sección áurea}

El problema de encontrar la sección áurea de un segmento dado aparece ya en los Elementos de Euclides donde se define, palabras más o palabras menos, de la siguiente manera:

Una línea recta se dice que se encuentra dividida en su razón media y extrema cuando la recta entera es al segmento mayor como el segmento mayor es al segmento menor.

En términos modernos:

Si se parte un segmento de recta en dos segmentos: uno grande de longitud $b$ y uno pequeño de magnitud $a$, se dice que éstos se encuentran en proporción áurea si

$$
\frac{a+b}{b}=\frac{b}{a}
$$

Es decir; si la razón del lado grande al lado chico es como la del total al lado grande. Como nos interesa la razón entre la longitud de los subsegmentos y no su magnitud, denotemos por $\phi$ al cociente $\frac{b}{a}$. De manera que la definición de sección, también llamada la proporción, implica que:

$$
\phi^{2}=\phi+1
$$

y resolviendo la ecuación cuadrática obtenemos dos soluciones:

$$
\phi_{1}=\frac{1+\sqrt{5}}{2} \quad \text { y } \quad \phi_{2}=\frac{1-\sqrt{5}}{2}
$$


La primera raíz, a la que de aquí en adelante llamaremos simplemente $\phi$ tiene un valor numérico aproximado de 1,618033988 ... se ve que la sucesión de dígitos después del punto decimal es infinita pues claramente se trata de un número irracional. Se deja al lector la demostración de que el valor de la segunda raíz es 0,618033988 . .

La proporción áurea tiene propiedades muy interesantes, entre ellas:

1. $\phi$ es el único número que se multiplica a sí mismo (se eleva al cuadrado) sumándose la unidad (ecuación 3). De aquí se desprende que $\phi^{3}=\phi^{2}+\phi$ y que sustituyendo $\phi+1$ en lugar de $\phi^{2}$ y ordenando los términos, se obtiene que $\phi^{3}=2 \phi=1$. Prosiguiendo de la misma manera, continuamos multiplicando ambos lados de la última igualdad por $\phi$ para obtener $\phi^{4}=3 \phi+2$ y así sucesivamente: $\phi^{5}=5 \phi+3, \phi^{6}=8 \phi+5$. En palabras: las potencias del número $\phi$ se expresan como un polinomio de primer grado cuyos coeficientes son elementos consecutivos de la sucesión de Fibonacci.

2. Despejando de $(3)$ se obtiene directamente que $\frac{1}{\phi}=\phi-1$. Es decir: $\phi$ es el único número cuyo inverso multiplicativo se obtiene restándose la unidad. Repitiendo los razonamientos del punto anterior, se tiene que: $\phi^{-1}=\phi-1$, multiplicando ambos lados por $\phi^{-1}$, se tiene que $\phi^{-2}=2-\phi$ y así sucesivamente: $\phi^{-3}=2 \phi-3, \phi^{-4}=5-3 \phi$ y pronto llegamos a la conclusión de que también las potencias negativas de $\phi$ se expresan como polinomios de primer orden cuyos coeficientes son números consecutivos de la sucesión de Fibonacci.

3. Una manera bonita de representar a $\phi$ es como una fracción continuada gracias al argumento de autosemejanza: como $\phi=1+\frac{1}{\phi}$ se tiene que:

$$
\phi=1+\frac{1}{1}+\frac{1}{1}+\frac{1}{1}+.
$$

El mismo razonamiento nos dice que como $\phi^{2}=1+\phi$, entonces $\phi=\sqrt{1+\phi}$ y de nuevo, razonando por autosemejanza:

$$
\phi=\sqrt{1+\sqrt{1+\sqrt{1+\sqrt{1+\sqrt{1+\ldots}}}}}
$$

La proporción áurea ha llamado la atención de muchos estudiosos a lo largo de la historia. Una lista completa de ellos no cabría aquí pero podemos mencionar a Luca Pacioli (15871576) pues escribió un libro que tuvo una gran influencia en las generaciones posteriores y cuyo título refleja el asombro casi místico que su propiedades le generaron: La proporción divina. Luca Pacioli fue un matemático italiano del renacimiento que contribuyó a la fundación de lo que hoy llamaríamos "matemáticas financieras". Fue maestro del gran Leonardo da Vinci y fue precisamente éste quien hizo las ilustraciones para su libro. 


\section{Fibonacci de oro}

Un hecho sorprendente pero no poco común de las matemáticas es la cercana relación que tienen entre sí fenómenos aparentemente lejanos. Este es el caso de la sección áurea y la sucesión de Fibonacci. Si evaluamos numéricamente la sucesión de cocientes de números consecutivos de Fibonacci, vamos a obtener:

$1 / 1=1,2 / 1=2,3 / 2=1,5 ; 5 / 3=1,666 \ldots ; 8 / 5=1,6,13 / 8=1,625,{ }^{21} / 13=1,6153 \ldots,{ }^{31} / 21=1,6190 \ldots$

que es una sucesión oscilante cuya razón entre términos consecutivos tiende a cero y que, por lo tanto, converge, y la conjetura, que daremos por cierta, es que:

$$
\lim _{n \rightarrow \infty} \frac{F_{n+1}}{F_{n}}=\phi
$$

En palabras: el cociente de términos sucesivos de la sucesión de Fibonacci converge a la sección áurea.

Regresemos a la expresión (2) y entonces tenemos que en el límite:

$$
\mathrm{F}=1+\frac{F n+1}{F_{n}}=1+\phi=\phi^{2}
$$

De nuevo, en palabras: El índice folial $\mathbb{F}$ de una planta es el cuadrado de la sección aúrea. Para redondear la discusión, consideramos que el índice folial de una planta se puede interpretar como el ángulo formado entre dos brotes consecutivos a lo largo de la parastiquia y la conclusión es que, siempre en el límite, el ángulo entre dos brotes es aproximadamente de 137,5 grados.

\section{El crecimiento áureo}

Entre los miles de resultados interesantes que nos legaron los antiguos griegos, se encuentra el concepto de gnomon. La siguiente definición es concisa y descriptiva:

Se dice que algo es el gnomon de una cosa, si es aquello que hay que agregarle para que siga siendo la misma cosa.

Antes de aventurar un ejemplo, recordemos que los griegos eran esencialmente geómetras y que para ellos un cuadrado era el polígono con cuatro lados iguales y cuyos ángulos internos eran todos rectos independientemente de su tamaño. Con esta motivación podemos ver que el gnomon de un cuadrado es una escuadra (figura 5).

Del mismo modo nos convencemos de que el gnomon de un disco es un anillo circular, el de un triángulo es un trapecio y podemos multiplicar los ejemplo hasta llegar a la pregunta 


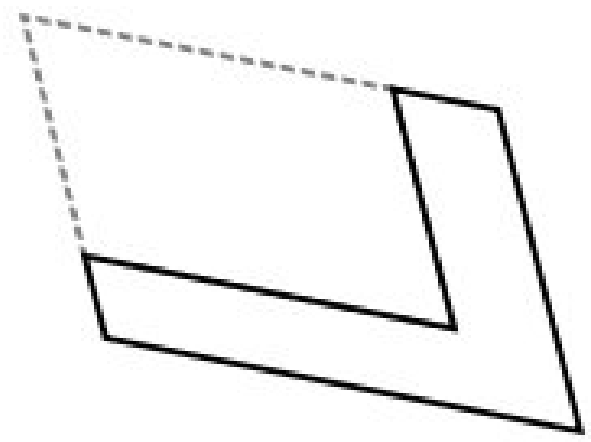

Figura 5: El gnomon de un cuadrado es una escuadra.

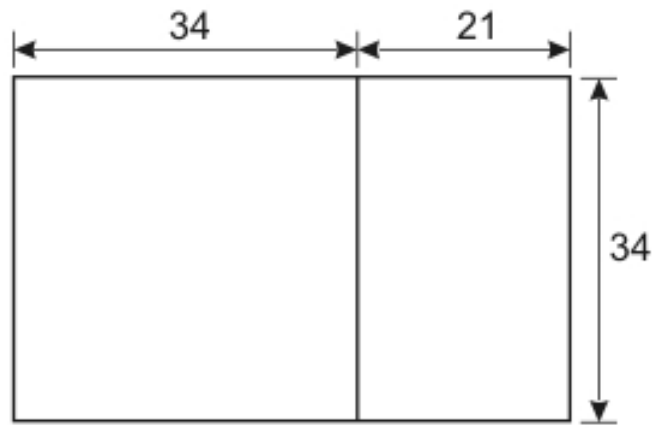

Figura 6: El rectángulo de 21 por 34 es aproximadamente áureo. Si se pega en su lado mayor un cuadrado de 34 por 34, la figura resultante es un rectángulo de 34 por 55 que también es aproximadamente áureo

natural: ¿Existe una figura que sea gnomon de sí misma? La respuesta no es sencilla. Para llegar a ella necesitamos el gnomon de un rectángulo áureo.

Se dice que un rectángulo es áureo si la razón entre sus lados va como la sección áurea. Gracias a nuestras aproximaciones numéricas de la proporción áurea, podemos afirmar que un rectángulo que mida trece por ocho es una aproximadamente áureo pero que el que mide veintiuno por trece, es mejor. El gnomon de un rectángulo áureo es un cuadrado que se le pega en uno de sus lados; digamos, el mayor. La figura 6 lo ilustra:

Si se repite el proceso de ir agregando los gnomones de los rectángulos aúreos tendremos la situación descrita en la figura 7a. Si se unen los vértices de los rectángulos áureos mediante una curva suave se obtiene una espiral logarítimica.

\section{Eadem mutata...}

Son tantas y tan asombrosas las propiedades de la espiral logarítmica que el gran Jakob Bernoulli no dudó en asentar en su testamento que en su lápida se grabase una espiral 


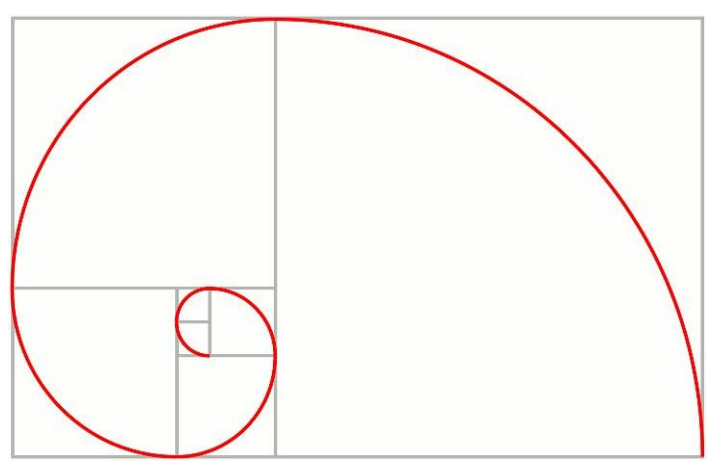

(a) Espiral logarítmica

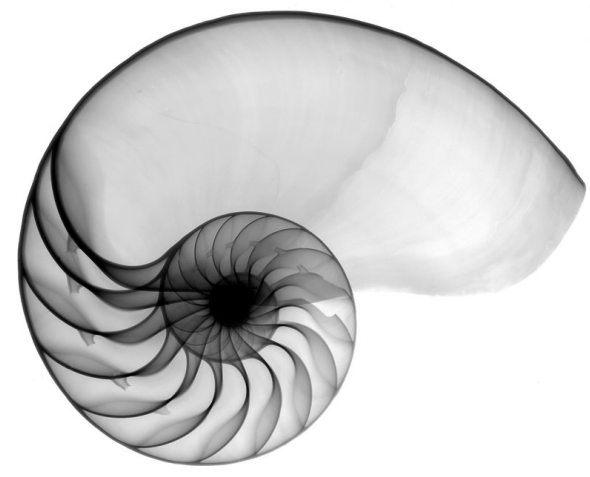

(b) Nautilus

Figura 7: Después de construir una sucesión de rectángulos áureos, se une con una curva continua sus vértices para obtener una espiral. En el lado derecho se muestra una imagen de rayos X del organismo Nautilus belauensis.

logarítmica ${ }^{1}$ con la leyenda Eadem mutata resurgo cuya traducción vendría siendo "aunque cambiada, resurjo la misma". La espiral logarítmica es la gráfica de la función exponencial en coordenadas polares. Como tal tiene la propiedad de que su tasa de crecimiento es ella misma. Una recta que pasa por el origen, intersecta la curva con el mismo ángulo no importando la orientación de la recta ni la rama de la espiral ${ }^{2}$. También se observan espirales logarítmicas en las ramas de las espirales galácticas, los nervios de la cornea y muchos otros lados.

En la foto del Nautilus (figura 7b) se aprecia claramente que la espiral logarítmica es la única figura en la naturaleza que es gnomon de sí misma. Se aprecia la estructura de cámaras o compartimientos en la concha del organismo y se ve que para que el mismo crezca, hay que agregar otra cámara que es un sector de espiral logarítmica es decir, un sector cuyas fronteras son ramas de espirales logarítmicas. Esto nos lleva a una afirmación muy contundente: Toda forma orgánica que crezca por acreción ${ }^{3}$ será por necesidad una espiral logarítmica. Esto se constata echando una mirada a caracoles, molúscos, cuernos de antílopes, etcétera.

Para los propósitos de este ensayo, se tiene que mencionar que formas espirales que aparecen en las plantas, como lo ilustran las figuras (2) son logarítmicas.

Hasta aquí hemos explorado algunas propiedades tanto de la sucesión de Fibonacci como de la sección áurea y hemos visto que guardan una estrecha relación. También hemos mostrado ejemplos de una gran cantidad de plantas en cuya arquitectura aparecen los conceptos anteriores. También hemos definido el índice folial de una planta para rematar con la presentación de la sucesión de Fibonacci en las plantas. Sin embargo, hasta aquí todo lo dicho

\footnotetext{
${ }^{1}$ Quiso la mala fortuna que el maestro cantero no supiese de matemáticas y grabara una espiral arquimedeana

${ }^{2}$ Esta es la razón por la cual un insecto se acerca a la llama de una vela siguiendo una trayectoria de espiral logarítmica pues el ojo compuesto del insecto, una vez que enfoca la fuente luminosa, guía al bicho sobre una trayectoria que se acerque a la luz pero manteniendo un ángulo constante con el radio.

${ }^{3}$ Agregando una capa lo ya existente.
} 
es una descripción de lo que ocurre en el mundo vivo, todo ha sido una narrativa atractiva o no dependiendo del gusto del lector. Pero todo lo expuesto no contiene una explicación causal de por qué son así las plantas. Una narrativa, por muy fina y detallada que sea, no es una explicación. Hemos hablado de la morfología de las plantas y ahora es momento de hablar de la morfogénesis de las mismas.

\section{Las matemáticas $\mathbf{y}$ las formas vivas}

La preocupación de llevar el estudio de las formas vivas al terreno de las matemáticas es antigua; poca gente sabe que el gran poeta Johan Wolfgang von Goethe, el autor de Fausto y de Las desventuras del joven Werther también tuvo una obra científica prolífica y que él consideraba como su obra maestra en este rubro al libro La metamorfosis de las plantas que si bien no contiene métodos matemáticos como los conocemos hoy en día, sí utiliza de manera pionera la noción de homología, tan apreciada tanto en la matemática como en las ciencias biológicas.

Posteriormente, en 1917, D’Arcy Wentworth Thompson obtuvo una cátedra de historia natural en la Universidad escocesa de Saint Andrews, misma que ocupó sesenta y cuatro años. Thompson nació en Edimburgo en el seno de una familia educada, su padre era profesor de griego, y se le reconoce hoy en día como el primer biomatemático moderno. Biólogo, matemático y estudioso de los clásicos grecolatinos, Thompson plasmó en su gran obra it On Growth and Form, una visión del mundo novedosa y revolucionaria que chocaría, y lo sigue haciendo, con el establishment de la biología. Thompson argumentó en el libro que los problemas de la forma de los seres vivos no se pueden entender sin el recurso de la matemática. En sus palabras:

Célula y tejido, concha y hueso, hoja y flor no son sino porciones de materia y el movimiento de sus partículas, su modelado y configuración ocurren en obediencia de las leyes físicas. No hay excepciones a la regla: Dios siempre hace geometría y sus problemas para dar forma son, en primera instancia, problemas matemáticos y sus problemas para definir el crecimiento, físicos. El morfólogo es, ipso facto, un estudiante de ciencias físicas.

Esta es, por razones obvias, quizá la cita más famosa y apreciada entre los estudiosos de la biología matemática.

Siguiendo esta línea de pensamiento, en 1992 dos investigadores franceses, S. Douady e Y. Couder publicaron un bellísimo trabajo titulado "La filotaxia como un proceso físico de crecimiento autoorganizado" [5] cuya lectura se recomienda enfáticamente. Sin embargo, en este ensayo presentaremos una metodología diferente con la misma intención.

\section{La emergencia de patrones}

Si se desea estudiar el origen de la forma en los seres vivos, hay que comenzar por preguntarse por la existencia de mecanismos generales que sean capaces de engendrar forma 
a partir de la homogeneidad. Cuando se tiene un medio homogéneo y uniforme y mediante algún cambio de valores de parámetros o por la acción de una fluctuación dinámica, el medio pierde su uniformidad y da lugar a formas geométricas discernibles, se dice, en lenguaje de los físicos, que ha ocurrido una ruptura de simetría. Uno de los pioneros en esta tarea fue el notable matemático inglés Alan M. Turing quién propuso un sistema de ecuaciones diferenciales parciales que desde entonces se llaman de "reacción-difusión". Supongamos que se tienen dos sustancias cuyas concentraciones denotamos por $u(x, y, t)$ y $v(x, y, t)$. Como lo indican sus variables independientes, las concentraciones de ambos compuestos dependen del tiempo y la posición en el plano. Supongamos también que los dos compuestos pueden reaccionar químicamente entre sí y que simultáneamente se difunden en el plano. Turing [4] propuso el siguiente sistema de ecuaciones diferenciales parciales:

$$
\begin{aligned}
& \left.\frac{\partial u}{\partial t}=D_{u} \Delta u+f(u, v)\right) \\
& \frac{\partial v}{\partial t}=D_{v} \Delta v+g(u, v)
\end{aligned}
$$

donde $D_{u}$ y $D_{v}$ son los coeficientes de difusión de los reactantes y $\Delta$ representa el operador laplaciano en dos dimensiones. Las funciones $f$ y $g$ son la parte correspondiente a la reacción o cinética como también suele llamársele.

Turing descubrió que si el sistema (5) en ausencia de difusión (el sistema se vuelve de ecuaciones diferenciales ordinarias) tiene un punto de equilibrio estable, aún cuando este punto sea estable, la acción de la difusión desestabiliza el sistema y, bajo ciertas condiciones sobre las funciones $f$ y $g$ y los coeficientes de difusión, se crean estados asintóticos estacionarios con concentración heterogénea de los compuestos. Es decir, la emergencia de patrones como un producto emergente del sistema mismo sin agentes externos. En la figura 8 se muestran algunos de estos patrones emergentes para cinéticas específicas y valores de los parámetros apropiados.

El interés de Turing por la filotaxia viene desde su infancia. En 1923 su madre dejó constancia de ello al hacer un dibujo en el que aparece su hijo Alan observando unas margaritas, mientras cinco compañeros suyos jugaban hockey. Varias décadas después, en sus archivos se encontró un dibujo en el que Turing contó las hileras de florecillas del girasol que, alineadas en forma de espiral: unas abren en el sentido del reloj y otras a contrarreloj (figura 9).

En los últimos años de su vida, a la luz de los estudios realizados por los hermanos Bravais en el que establecen una conexión insospechada entre la cristalografía y la botánica ${ }^{4}$, Turing extiende la filotaxia geométrica de éstos y planteó lo que él mismo llamó teoría dinámica de la filotaxia. En ella, el británico tomó como reto escribir en forma matemática los procesos subyacentes a la emergencia de los brotes a lo largo del tallo de las plantas. En tres trabajos publicados póstumamente Turing dejó su legado sobre filotaxia.

En el siguiente mensaje enviado a su colega C.W. Wardlaw:

\footnotetext{
${ }^{4}$ La idea es simple: al poner una marca (un punto) en cada uno de los brotes sobre el tallo de la planta en cuestión, y se desenrrolla la corteza, lo que se obtiene es una superficie y sobre ella una retícula cuyos puntos, al unirse, forman las parastiquias.
} 


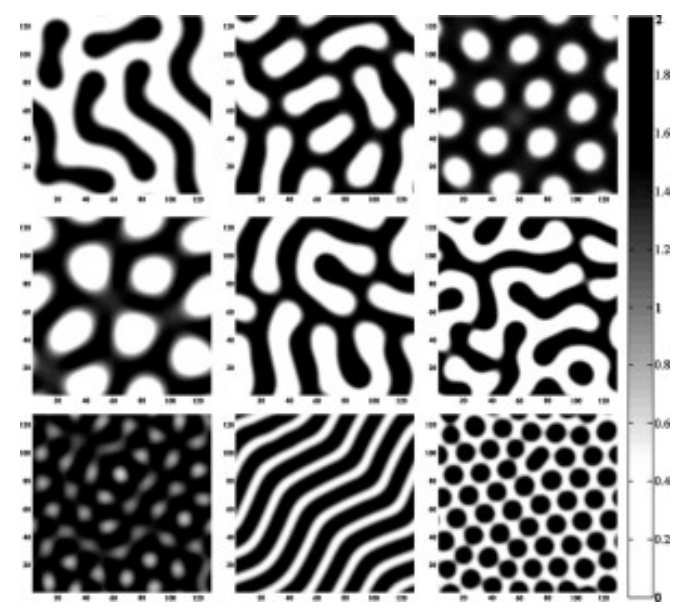

Figura 8: Soluciones estacionarias de las ecuaciones de reacción-difusión de Turing con diferentes juegos de parámetros.
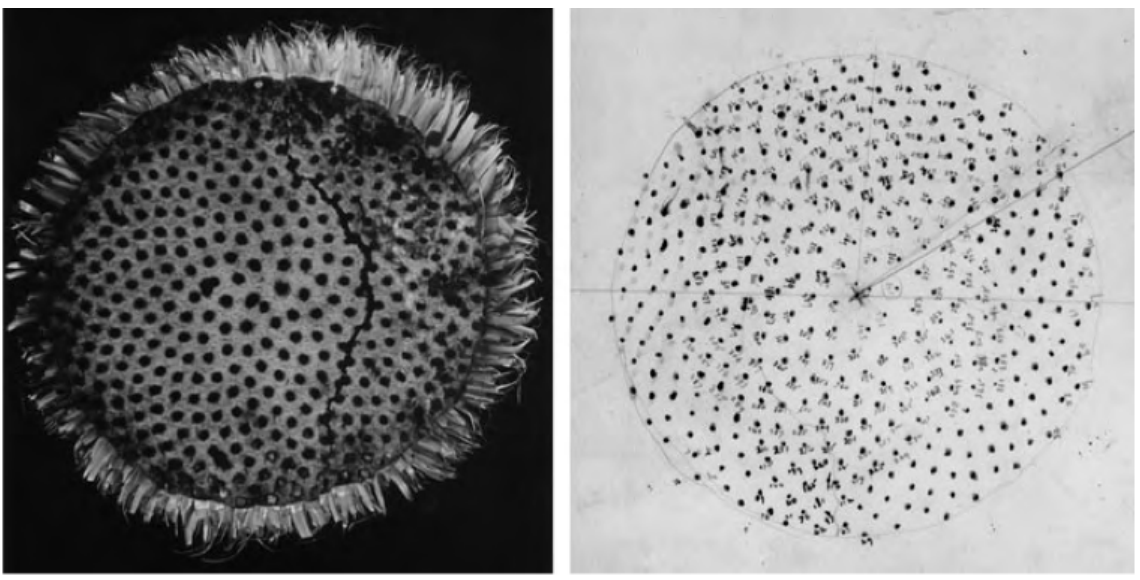

Figura 9: Dibujo realizado por Turing en el que se muestran las hileras de florecillas del girasol.

Nuestra nueva máquina ${ }^{5}$ llegará el lunes. Tengo la esperanza de que uno de los primeros trabajos sea hacer algo sobre "la embriología química". En particular, creo poder responder a la pregunta sobre la aparición de los números de Fibonacci en las piñas de los abetos.

queda claro que Turing tenía la ilusión de entender el mecanismo que genera las filotaxias. Desafortunadamente, fue un proyecto que ya no pudo desarrollar por el final abrupto que tuvo su vida y que todos conocemos.

\footnotetext{
${ }^{5}$ La Ferranti Mark I, primera computadora comercial de uso general
} 

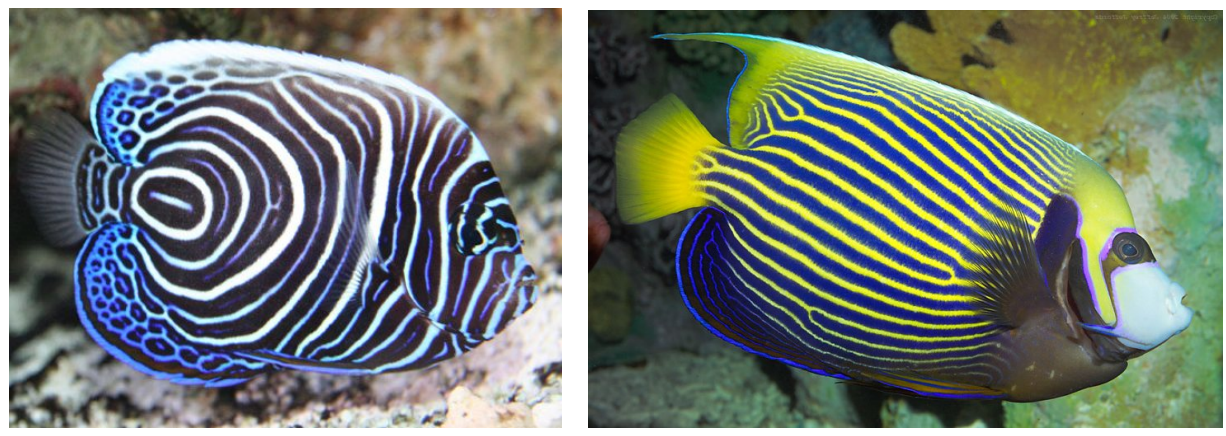

Figura 10: El pez Pomacanthus imperator en sus etapas juvenil y adulta

\section{Emergencia de patrones en dominios con crecimiento}

En los últimos lustros se ha dado un creciente interés por el estudio del efecto que tienen en la selección de patrones el crecimiento y la curvatura del dominio en el que los procesos básicos de reacción y difusión se llevan a cabo. El problema reviste particular importancia por el hecho de existir organismos en los cuales ambos factores influyen de forma determinante en la emergencia de patrones. Sin ir más lejos, los patrones de coloración que exhiben los peces del género Pomacanthus imperator, son especialmente interesantes: cuando éstos son juveniles presentan patrones de coloración en forma de franjas que corren a lo largo de su cuerpo, pero a medida que crecen — además de que el número de franjas aumenta - llega a ocurrir que la orientación de éstas cambia drásticamente: de ser transversales a longitudinales (figura 10) y todo ello por el crecimiento del cuerpo (. ${ }^{\mathrm{El}}$ dominio", diría un matemático) aunque la dinámica, metabolismo y compuestos sean los mismos.

Recordemos que el propósito de este ensayo es hablar de los saguaros. Arriba se argumentó que su estructura costillar es semejante a la de las biznagas globulares. En esta sección retomamos la arquitectura de las estrías que presenta una especie de biznaga y trataremos de dilucidar los mecanismos que hacen posible su disposición espacial. Toda la matemática aquí hecha se ha elaborado sobre dominios esféricos. Posiblemente se obtengan resultados semejantes para el caso específico de los saguaros sobre dominios cilíndricos. Para este tratamiento usaremos un marco teórico general propuesto por Plaza et al [6]. En esta referencia, a partir de primeros principios, sus autores dedujeron un sistema de ecuaciones diferenciales parciales de tipo reacción-difusión el cual incorpora tanto el crecimiento, como la curvatura del dominio en el que los patrones emergen. No es este el lugar para reproducir los detalles técnicos, baste decir que el sistema de reacción-difusión al que se llega en vez de aparecer el operador de Laplace mencionado en el sistema 5, aparece el operador de Laplace-Beltrami ${ }^{6}$.

La geometría de la biznaga es aproximadamente esférica y sobre su superficie aparecen estrías verticales - como los meridianos terrestres - y en éstas se encuentran los nodos de espinas. También hay biznagas cuyas estrías exhiben una organización espacial en forma de espiral. Para los fines de esta exposición, supondremos además que la biznaga crece

\footnotetext{
${ }^{6}$ Esta es la versión que toma el Laplaciano sobre variedades de dimensión dos contenidas en $\mathbf{R}^{3}$.
} 
isotrópicamente. Esta forma de crecer mantiene las proporciones de las diferentes dimensiones de la biznaga a medida que su tamaño aumenta por lo que su aspecto se mantiene a medida que aumenta de tamaño.

\subsection{Filotaxia de la biznaga globosa}

Siguiendo el planteamiento desarrollado en Plaza et al. [6], tomemos como dominio a una esfera parametrizada:

$$
X(\xi, \eta, t) \equiv \rho(t)\left(\begin{array}{c}
\operatorname{sen} \eta \cos \xi \\
\operatorname{sen} \eta \operatorname{sen} \xi \\
\cos \eta
\end{array}\right)
$$

donde $\xi \in[0,2 \pi], \eta \in[0, \pi]$, y tomando

$$
h_{1}^{2}=\left|X_{\xi}\right|^{2}=\rho^{2} \operatorname{sen}^{2} \eta, \quad h_{2}^{2}=\left|X_{\eta}\right|^{2}=\rho^{2}, \text { de donde } \frac{h_{1}}{h_{2}}=\operatorname{sen} \eta,
$$

se llega al sistema que describe a los procesos de reacción y difusión sobre la geometría mencionada

$$
\begin{gathered}
u_{t}=\frac{1}{\rho^{2}}\left(u_{\eta \eta}+\frac{1}{\operatorname{sen}^{2} \eta} u_{\xi \xi}-\frac{\cos \eta}{\operatorname{sen} \eta} u_{\eta}\right)-\frac{2 \dot{\rho}}{\rho} u+f(u, v), \\
v_{t}=\frac{d}{\rho^{2}}\left(v_{\eta \eta}+\frac{1}{\operatorname{sen}^{2} \eta} v_{\xi \xi}-\frac{\cos \eta}{\operatorname{sen} \eta} v_{\eta}\right)-\frac{2 \dot{\rho}}{\rho} v+g(u, v) .
\end{gathered}
$$

donde $u_{t}$ y $v_{t}$ representan respectivamente las derivadas parciales temporales de las variables $u=u(x, y, t)$ y $v=v(x, y, t)$

Si como supusimos, el crecimiento es isotrópico con función de crecimiento $\rho(t)=\exp (k t)$, entonces el sistema (7) toma la forma

$$
\begin{aligned}
& u_{t}=\frac{1}{e^{2 k t}}\left(u_{\eta \eta}+\frac{1}{\operatorname{sen}^{2} \eta} u_{\xi \xi}-\frac{\cos \eta}{\operatorname{sen} \eta} u_{\eta}\right)-2 k u+f(u, v), \\
& v_{t}=\frac{d}{e^{2 k t}}\left(v_{\eta \eta}+\frac{1}{\operatorname{sen}^{2} \eta} v_{\xi \xi}-\frac{\cos \eta}{\operatorname{sen} \eta} v_{\eta}\right)-2 k v+g(u, v) .
\end{aligned}
$$

La parte reactiva que consideramos es de tipo FitzHugh-Nagumo que, como es sabido, describe sistemas que exhiben excitabilidad. Luego, las ecuaciones (8) se escriben como 


$$
\begin{aligned}
& u_{t}=\frac{1}{e^{2 k t}}\left(u_{\eta \eta}+\frac{1}{\operatorname{sen}^{2} \eta} u_{\xi \xi}-\frac{\cos \eta}{\operatorname{sen} \eta} u_{\eta}\right)-2 k u+c\left(u-\frac{u^{3}}{3}+v+z_{0}\right), \\
& v_{t}=\frac{d}{e^{2 k t}}\left(v_{\eta \eta}+\frac{1}{\operatorname{sen}^{2} \eta} v_{\xi \xi}-\frac{\cos \eta}{\operatorname{sen} \eta} v_{\eta}\right)-2 k v-\frac{u-a+b v}{c} .
\end{aligned}
$$

9.2. Simulaciones numéricas

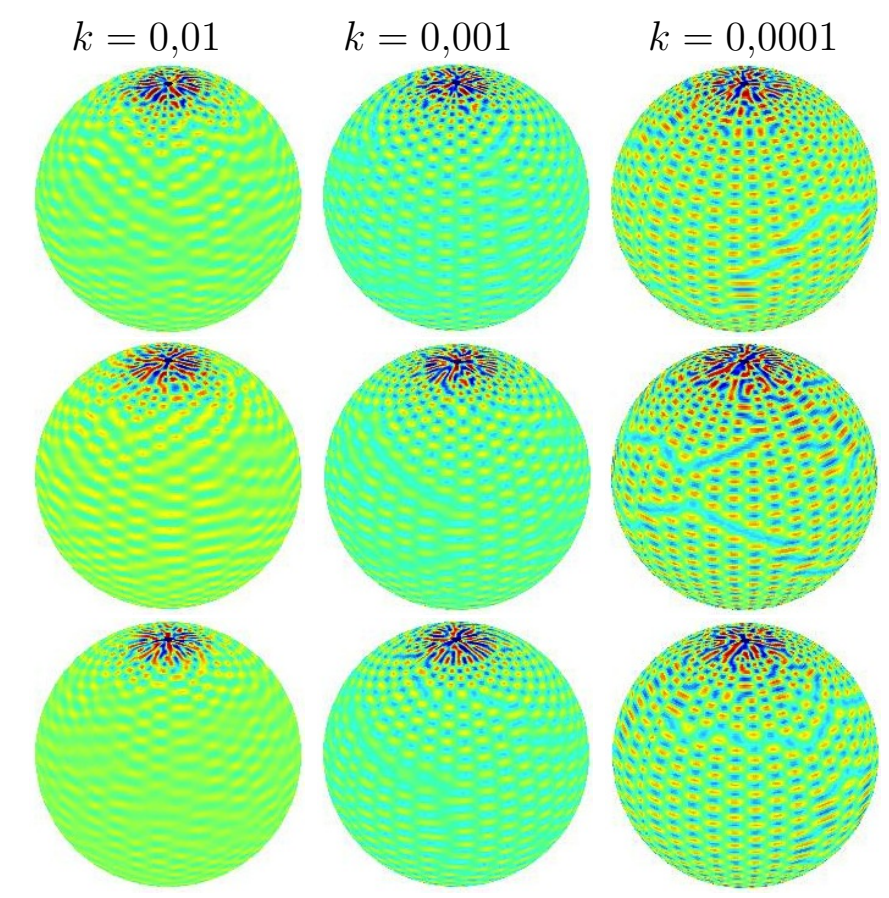

Figura 11: Simulaciones de la biznaga a distintas tasas de crecimiento, mismas que aparecen arriba de cada columna. En la primera columna las imágenes son tomadas a las 10000 iteraciones; en la segunda columna las imágenes son tomadas a las 80000 iteraciones; mientras que en la tercera columna son tomadas a las 600000 iteraciones.

En las simulaciones numéricas que se muestran - que se reportan en [3] - en la figura 11 aparece la concentración de $u$ y se realizaron usando el siguiente juego de valores de los parámetros: $z=-0,6, a=0,5, b=0,8, c=0,89, d=6,0$.

El punto de equilibrio positivo obtenido en cada fila es fijado y se aumenta o disminuye la tasa de crecimiento como se detalla a continuación.

En la primera fila fijando la tasa de crecimiento en $k=0,01$ el punto singular obtenido es $u_{0}=0,04633, v_{0}=0,55474$. La imagen en la segunda columna es obtenida al disminuir la tasa de crecimiento a $k=0,001$. Para la tercera columna la tasa de crecimiento es disminuida a $k=0,0001$. 

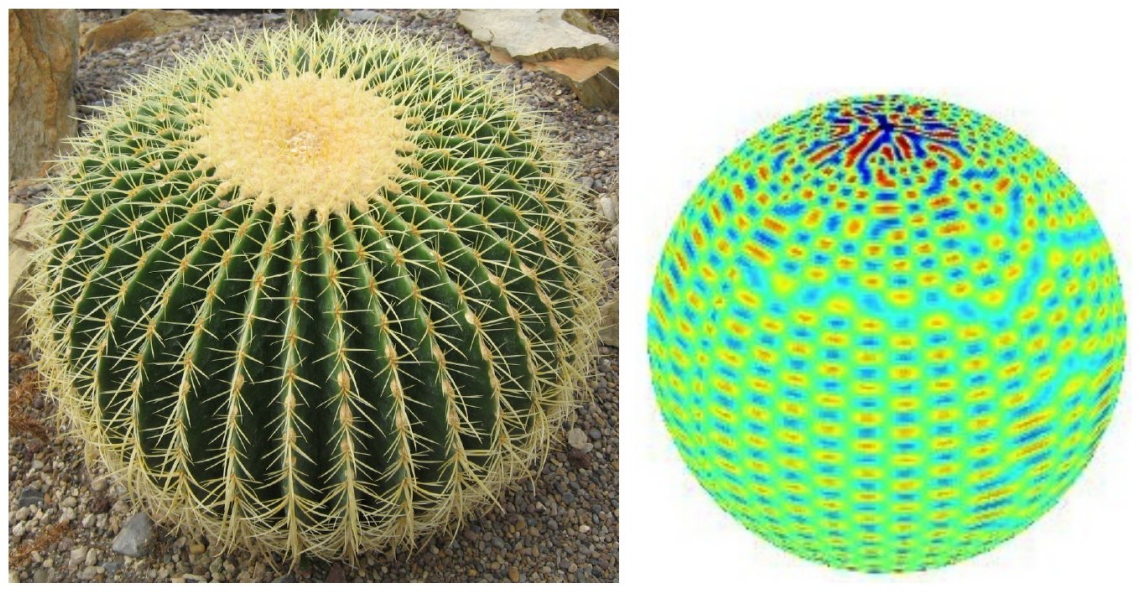

Figura 12: A la izquierda tenemos una biznaga globular y la derecha su modelo matemático

En la segunda fila el punto de equilibrio es $u_{0}=0,09356, v_{0}=0,50692$, el que está asociado a la tasa de crecimiento $k=0,001$. La imagen en la primera columna es obtenida al aumentar la tasa de crecimiento a $k=0,01$, mientras que la imagen en la tercera columna es obtenida al tomar $k=0,0001$.

Por último, en la tercera fila el punto singular es $u_{0}=0,09820, v_{0}=0,50213$, el cual es calculado cuando $k=0,0001$. La imagen en la segunda columna es obtenida al aumentar la tasa de crecimiento a $k=0,001$ y finalmente la imagen en la primera columna es obtenida al considerar $k=0,01$.

\section{Conclusiones}

Entender los mecanismos que generan la forma de los organismos es una tarea fundamental de la ciencia. Como se ha mostrado en este ensayo, hay avances notables pero aún quedan muchos problemas por resolver. Bajo el nombre genérico de morfogénesis se agrupa un conjunto enorme de metodologías, teorías y herramientas matemáticas que intentan dar explicaciones causales al desarrollo y evolución de las formas vivas. La biología y la matemática se enriquecen mutuamente: las ecuaciones diferenciales parciales de reacción-difusión han tenido un avance espectacular desde la década de los cincuenta del siglo pasado y cuesta trabajo imaginar si ese desarrollo hubiera sido igual de no haber sido por su motivación en la morfogénesis. Por otra parte, Turing postuló la existencia de hipotéticos morfógenos cuya concentración inhomogénea daría lugar a las rupturas de simetría durante el desarrollo embrionario. Esta sugerencia motivó a la comunidad biológica a buscar dichos compuestos y hoy en día ya se conocen algunas fitohormonas que cumplen ese papel. Un modelo matemático es una representación imperfecta de la realidad pero cuando su resultado se asemeja tanto a ésta (figura 12 entonces juega un papel muy relevante en la comprensión del fenómeno pues al recoger de manera tan buena los aspectos más relevantes del mismo, se pueden variar los parámetros y observar lo que sucede con el modelo y, eventualmente, hacer predicciones del fenómeno estudiado. 
Evidentemente, para resolver un problema biológico bajo la perspectiva química o fisicalista es necesario un lenguaje que articule la comunicación entre las disciplinas y ese lenguaje es la matemática.

\section{Agradecimientos}

PM desea agradecer el apoyo de la PASPA/DGAPA y al proyecto PAPIIT IN107414. 


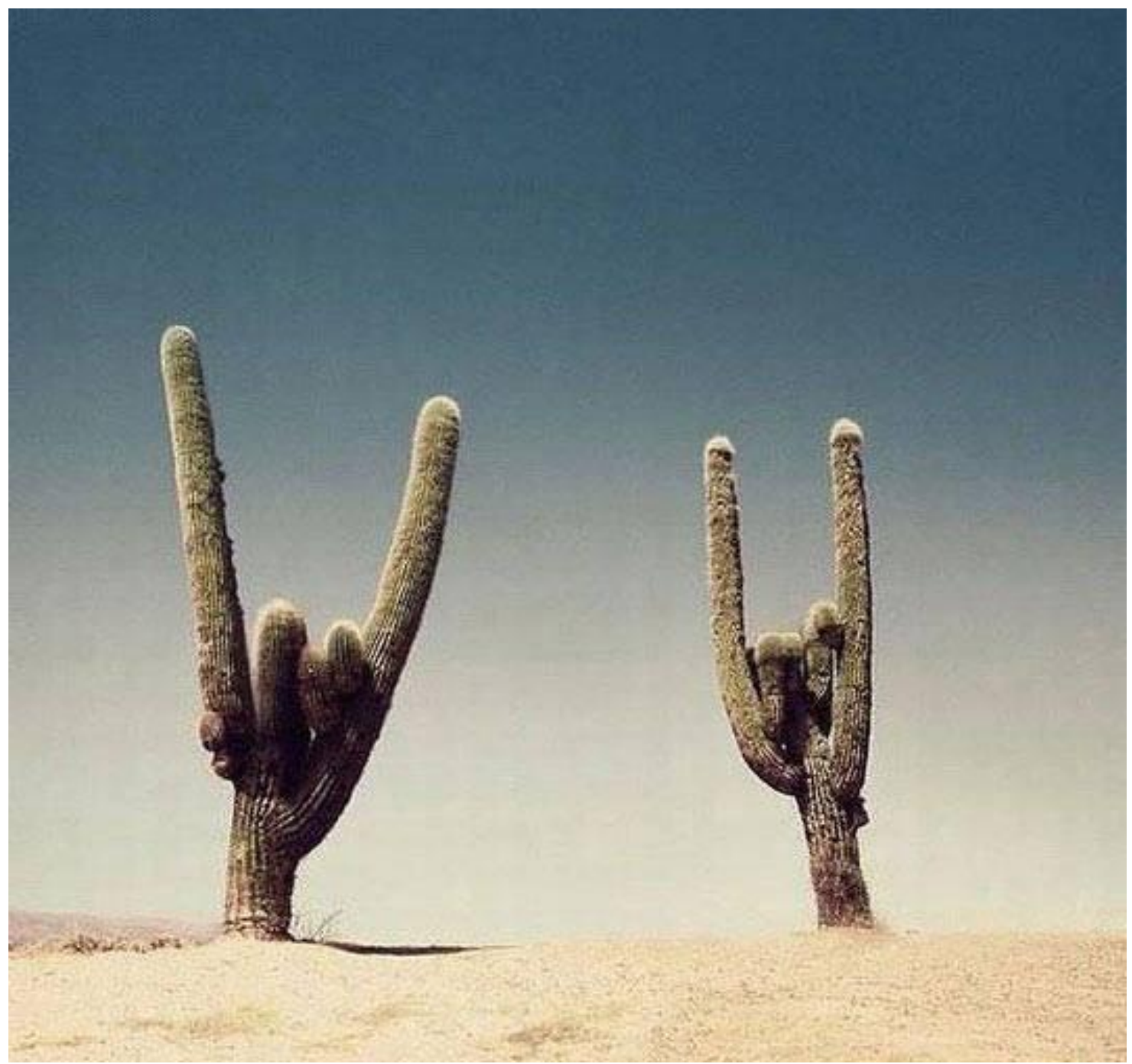

\section{Referencias}

[1] D’Arcy W. Thompson, On Growth and Form, Dover Publications, NY. 1992.

[2] J.A. Castillo Medina, F. Sánchez Garduño y P. Padilla Longoria: De las formas de las naturaleza a las matemáticas. Ciencia, Vol. 64, No. 4 pp. 64-71, 2013.

[3] J.A. Castillo Medina: Morfogénesis en superficies dinámicas. Tesis doctoral. Coordinación de Estudios de Posgrado. Programa de Maestría y Doctorado en Ciencias Matemáticas, UNAM, 2015.

[4] Turing, A. M. The Chemical Basis of Morphogenesis. Philosophical Transactions of the Royal Society of London 237 (641): 37 ?72 (1952), 
[5] S. Douady and Y. Couder: Phyllotaxis as a physical self-organized growth process. Physical Review Letters. 68, 2098. 1992

[6] Ramón Plaza, Faustino Sánchez-Garduño, Pablo Padilla, Rafael Barrio and Philip K. Maini: The effect of growth and curvature on pattern formation. Journal of Dynamics and Differential Equations, Vol. 16, No. 4, October, pp. 1093-1121, 2004. 